\title{
Short- and long-term impact of vaccination against cytomegalovirus: a modeling study
}

\author{
Ganna Rozhnova1,2,4* (D), Mirjam E. Kretzschmar ${ }^{1,2}$, Fiona van der Klis², Debbie van Baarle ${ }^{2,5}$, \\ Marjolein J. Korndewal ${ }^{2}$, Ann C. Vossen ${ }^{3}$ and Michiel van Boven ${ }^{2}$
}

\begin{abstract}
Background: Infection with cytomegalovirus (CMV) is highly prevalent worldwide and can cause severe disease in immunocompromised persons and congenitally infected infants. The disease burden caused by congenital CMV infection is high, especially in resource-limited countries. Vaccines are currently under development for various target groups.
\end{abstract}

Methods: We evaluated the impact of vaccination strategies and hygiene intervention using transmission models. Model parameters were estimated from a cross-sectional serological population study $(n=5179)$ and a retrospective birth cohort $(n=31,484)$, providing information on the age- and sex-specific CMV prevalence and on the birth prevalence of congenital CMV (CCMV).

Results: The analyses show that vertical transmission and infectious reactivation are the main drivers of transmission. Vaccination strategies aimed at reducing transmission from mother to child (vaccinating pregnant women or women of reproductive age) can yield substantial reductions of cCMV in 20 years (31.7-71.4\% if 70\% of women are effectively vaccinated). Alternatively, hygiene intervention aimed at preventing CMV infection and re-infection of women of reproductive age from young children is expected to reduce cCMV by less than $2 \%$. The effects of large-scale vaccination on CMV prevalence can be substantial, owing to the moderate transmissibility of CMV at the population level. However, as CMV causes lifelong infection, the timescale on which reductions in CMV prevalence are expected is in the order of several decades. Elimination of CMV infection in the long run is only feasible for a vaccine with a long duration of protection and high vaccination coverage.

Conclusions: Vaccination is an effective intervention to reduce the birth prevalence of cCMV. Population-level reductions in CMV prevalence can only be achieved on a long timescale. Our results stress the value of vaccinating pregnant women and women of childbearing age and provide support for the development of CMV vaccines and early planning of vaccination scenarios and rollouts.

Keywords: Cytomegalovirus, CMV, Congenital CMV infection, Transmission model, Vaccination strategies, Hygiene intervention, CMV elimination, CMV burden

\footnotetext{
*Correspondence: G.Rozhnova@umcutrecht.nl

1 Julius Center for Health Sciences and Primary Care, University Medical Center

Utrecht, Utrecht University, Utrecht, The Netherlands

${ }^{2}$ Center for Infectious Disease Control, National Institute for Public Health and

the Environment, Bilthoven, The Netherlands

Full list of author information is available at the end of the article
}

(C) The Author(s). 2020 Open Access This article is licensed under a Creative Commons Attribution 4.0 International License, which permits use, sharing, adaptation, distribution and reproduction in any medium or format, as long as you give appropriate credit to the original author(s) and the source, provide a link to the Creative Commons licence, and indicate if changes were made. The images or other third party material in this article are included in the article's Creative Commons licence, unless indicated otherwise in a credit line to the material. If material is not included in the article's Creative Commons licence and your intended use is not permitted by statutory regulation or exceeds the permitted use, you will need to obtain permission directly from the copyright holder. To view a copy of this licence, visit http://creativecommons.org/licenses/by/4.0/. The Creative Commons Public Domain Dedication waiver (http://creativecommons.org/publicdomain/zero/1.0/) applies to the data made available in this article, unless otherwise stated in a credit line to the data. 


\section{Background}

Human cytomegalovirus (CMV) is a highly prevalent herpesvirus with lifetime probability of infection that ranges from 50 to $100 \%$ in populations throughout the world [1]. Primary infection is usually mild or even asymptomatic. However, in infants with congenital CMV infection (cCMV) and in recipients of solid organ and stem cell transplants, the risk of severe disease is high [2-5]. Moreover, CMV has been linked to low-grade inflammation, atherosclerosis, and associated diseases [6]; requires considerable resources of the host's immune system [7, 8]; and could negatively affect the effectiveness of vaccination against other pathogens in elderly [9].

The burden of disease caused by CMV infection worldwide is high. In the USA, an estimated 7000 children per year are affected by disease caused by cCMV, which is more than for other congenital disorders [10]. In a systematic review of studies in developing countries, Lanzieri et al. [11] found that maternal seroprevalence ranged from 84 to $100 \%$ and CMV birth prevalence from 0.6 to $6.1 \%$, which is considerably higher than rates reported for Europe and North America [12]. Estimates of disabilityadjusted life years (DALYs) due to cCMV are scarce. In a study from Belgium, the burden of disease due to $\mathrm{CCMV}$ was estimated at 18 DALYs per 100,000 in Belgium for 2013, and ten times higher than the burden caused by congenital toxoplasmosis [13]. An estimated 25\% of cases of hearing loss in young children in the USA has been attributed to cCMV [14]. The high prevalence of maternal CMV infection and incidence of cCMV worldwide $[3,15-18]$ has motivated the development of vaccines $[19,20]$. No vaccine is as yet registered, but several candidate vaccines are well past the stage of early development and are being evaluated in clinical trials [20-22]. Another preventive option to reduce $\mathrm{cCMV}$ is hygiene intervention [23-26], whose effectiveness among seronegative pregnant women with exposure to young children has been supported by several studies [23, 25].

The transmission of CMV from an infected to uninfected host can occur in direct contact [27] (mediated by urine or saliva), from mother to child during pregnancy $[28,29]$, or via breastfeeding [3, 30, 31]. In addition, direct transmission does occur not only during primary infection of a newly infected host, but also after reactivation of the virus or re-infection in a latently infected host [32]. Hence, the transmission dynamics of CMV is complex, and there is at best a partial understanding of the quantitative contributions of each of the transmission routes to the epidemiology of CMV. Such a quantitative insight is essential for proper evaluation of the impact of interventions. We take a step in this direction, by evaluating vaccination strategies and hygiene intervention with a transmission model calibrated to two large population studies from the Netherlands [33, 34].
Modeling studies on the CMV transmission dynamics are scarce. Previous studies focused on the costeffectiveness of CMV vaccination $[35,36]$ or evaluated the impact of vaccination on reducing $\mathrm{CCMV}$ with transmission models [37-40]. However, these models did not include all known transmission routes (e.g., reactivation and multiple re-infections [37, 40]). Furthermore, the potential impact of vaccinating pregnant women and hygiene intervention aimed at preventing CMV (re-) infection of women of reproductive age from young children [25] has not been assessed. In addition, most models were parameterized using CMV seroprevalence among the population from the USA, not stratified by sex [38-40], which in some studies was limited to persons with ages between 0 and 49 years $[38,40]$. Due to uncertainties in key epidemiological parameters, predictions of these models vary widely.

Our model includes direct transmission after primary infection, re-infection, or reactivation, and vertical transmission (congenitally and postnatally, defined here as transmission in the first 6 months of life, mainly via breastfeeding). The model is an extension of an earlier developed model, allowing for multiple lifetime re-infection and reactivation events [41]. To our knowledge, this is the first model to include all known transmission routes. The model parameters are estimated using data from a birth cohort and a large population-based serological study among persons with a wide age range ( $0-80$ years) $[33,34]$. Based on quantitative estimates of key parameters, we evaluate the effectiveness of a suite of vaccination strategies and hygiene intervention decreasing infectious contacts of women of reproductive age and young children. Specifically, we consider vaccination during pregnancy and several population-wide vaccination strategies (in children, adolescents, or adults; women only vs men and women), using varying vaccination coverages and durations of protection after vaccination.

We report results on the reduction in the birth prevalence of cCMV and prevented disease burden within 20 years of the start of interventions. We also provide insight into whether or not the elimination of CMV from the population is possible for each intervention. Finally, we discuss the implications of our findings for future implementation of interventions.

\section{Methods}

\section{Overview}

Estimates of epidemiological parameters were obtained by fitting a transmission model to data from (1) a crosssectional population-based serological study proving ageand sex-specific seroprevalence $(n=5179)$ [33] and (2) a nation-wide retrospective birth cohort study providing the birth prevalence of $\mathrm{cCMV}(n=31,484)$, both from the Netherlands $[34,42]$. The model, equipped with 
parameters estimated from these two studies, was subsequently used to investigate the effectiveness of different interventions.

\section{Data}

The cross-sectional population-based serological study was carried out in the Netherlands in 2006 and 2007 [33, 43]. A total of 40 out of 467 municipalities were randomly selected with probabilities proportional to their population size. From these municipalities, an agestratified sample was drawn from the population register, and 19,781 persons were invited to complete a questionnaire and to donate a blood sample. Serum samples and questionnaires were obtained from 6382 participants. We excluded infants younger than 6 months to avoid interference with maternal antibodies and non-western migrants to preclude confounding by ethnicity. The final sample $(n=5179)$ included 2842 women and 2337 men.

In the retrospective birth cohort, children born in the Netherlands between 1 January 2008 and 1 October 2008 were eligible for entry [34, 42]. Informed consent for retrieving the dried blood spot sample taken shortly after birth was obtained from 31,484 participants. Of children tested during the study period, $154(0.5 \%)$ were confirmed positive by CMV DNA PCR. The birth cohort initially had been set up to study the long-term sequelae and disease burden of cCMV [34, 42].

Our analyses made use of demographic composition and fertility rates in 2017 from Statistics Netherlands (www.cbs.nl) and age- and sex-specific contact data [41, 44].

\section{Transmission model}

We developed a deterministic compartmental model describing CMV transmission in a population stratified by CMV infection status, sex, and age (Fig. 1). The model includes direct transmission from persons who experience a primary infection, a re-infection, or a reactivation episode and vertical transmission (congenital and postnatal via breastfeeding). Persons are classified as seronegative (susceptible, $S$ ) and seropositive who can be latently infected with low (latent, $L$ ) or with high antibody concentrations (boosted, $B$ ), or acutely infected in three infectious classes $\left(I_{1}\right.$ to $\left.I_{3}\right)$ corresponding to primary infection $\left(I_{1}\right)$, and re-infection or reactivation from the $L$ and $B$ class $\left(I_{2}\right.$ and $\left.I_{3}\right)$. The force of infection, $\lambda$, and reactivation rate, $\rho$, are age- and sexspecific (not shown). The force of infection is given by a weighted sum of the fraction of the population in the three infectious classes. Latently infected persons can be re-infected at a rate $z \lambda$, where $z$ is the reduction in susceptibility to re-infection in latently infected persons compared to seronegative persons. Vertical transmission occurs with probability $q$ from seropositive women (classes $L, B, I_{1}, I_{2}$, and $I_{3}$ ). Congenital transmission occurs with probability $q_{c}$ from acutely infected women $\left(I_{1}, I_{2}\right.$, and $\left.I_{3}\right)$.

\section{Parameter estimation}

Estimation of model parameters followed a previous study $[41,45]$. Here, we extended the earlier analyses by (i) allowing for multiple reactivation and re-infection events occurring over a person's life, (ii) using cubic B-splines for flexible estimation of the age-dependent reactivation rates, and (iii) including the birth cohort data to enable estimation of the probability of cCMV. For horizontal transmission, we used an age- and sex-specific contact matrix with 17 age classes [41, 44]. The model is fitted to the data using the Hamiltonian Monte Carlo method as

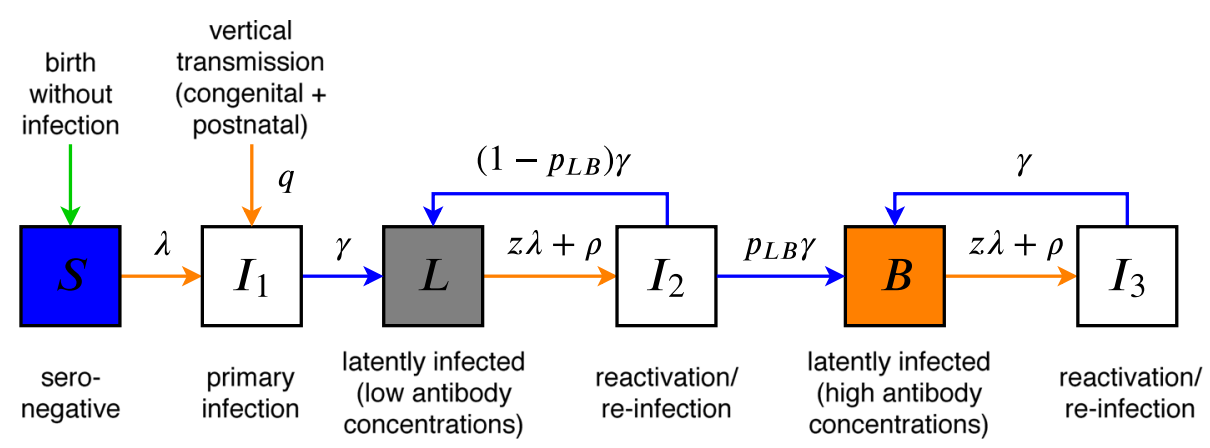

Fig. 1 Schematic of the transmission model. Shown are epidemiological transitions in the transmission model without vaccination. Seronegative persons (susceptible, S) acquire primary infection $\left(l_{1}\right)$ either vertically from their seropositive mothers (with probability $q$ ) or horizontally (with the force of infection $\lambda$ ) via contact with acutely infected persons in three infectious classes $\left(/ I_{1}, I_{2}, I_{3}\right)$. After primary infection (duration $1 / \gamma$ year), persons become latently infected with low antibody concentrations (latent, $L$ ). In this class, re-infection occurs at a rate $z \lambda$, where $z$ is the reduction in susceptibility to re-infection in latently infected persons compared to seronegative persons, and reactivation occurs at a rate $\rho$. After reactivation/re-infection (duration $1 / \gamma$ year), persons transit to the latent class with high antibody concentrations (boosted, $B$ ) with probability $p_{L B}$, where further re-infection and reactivation events can occur $(/ 3)$. Note that vertical transmission from mother to child includes both congenital and postnatal transmission via breastfeeding. The population is stratified by sex and age, and the forces of infection and reactivation rates are age- and sex-specific (not shown). Figure S1 and Figure S2 give schematics of the full model with vaccination 
implemented in Stan (www.mc-stan.org) [46]. Details are given in Additional file 1: Appendix.

\section{Intervention scenarios}

We considered several intervention strategies [20, 23-26, 47], namely hygiene measures [23, 25] aimed at preventing CMV (re-)infection of women of reproductive age from young children, vaccination during pregnancy reducing the probability of vertical transmission, and a suite of universal vaccination strategies $[20,47]$ with varying proportions of effectively vaccinated persons, ages at vaccination, sexes to be vaccinated, and durations of protection.

For universal vaccination, we further distinguished between scenarios in which the vaccine is assumed to protect only against primary infection in seronegative persons ("prevention of infection") or against primary infection in seronegative persons and re-infection/reactivation in seropositive persons ("prevention of (re-)infection and reactivation") $[20,47]$. The target population for vaccination was either infants in the first year of life, adolescent boys and girls at the age of 10 years, adolescent girls at the age of 10 years, or women of reproductive age (15-50 years).

In the baseline scenario, we assumed that the proportion of effectively vaccinated persons (henceforth called effectively vaccinated proportion $=$ vaccination coverage $\times$ vaccine efficacy) was $70 \%$. The average duration of protection by the vaccine was 10 years. Hygiene measures assumed a $70 \%$ reduction in infectious contacts between 15-50-year-old women and 0-5-year-old children.

Main outcome measures were the reduction in the incidence of cCMV, primary infection and reinfection/reactivation, and DALYs prevented after 20 years. In addition, we evaluated the long-term impact of an intervention by computing the effective reproduction number and (critical) proportion of persons who must be effectively vaccinated to eliminate CMV from the population $[45,48,49]$. The effective reproduction number quantifies the intervention effort necessary for disease elimination. In theory, a disease is eliminated whenever this number is below $1[45,48]$.

The model was implemented using a system of ordinary differential equations for 165 -year age groups and a group of 0-6-month-old infants (Additional File 1: Appendix). All interventions were introduced from the endemic equilibrium. The disease burden for the Netherlands was computed using an estimate of 3.034 (95\%CrI 1.202-6.105) DALYs per case of cCMV [13].

\section{Sensitivity analyses}

We conducted sensitivity analyses for the effectively vaccinated proportion and duration of protection after vaccination. Specifically, we varied the proportion from 0 to $100 \%$ and the duration of protection from 2.5 years to lifelong. In addition, we present parameter estimation and intervention results for a different set of prior distributions of the reactivation rates (Figure S4, Figure S5 and Table S2).

\section{Results}

\section{Parameter estimation}

Figure 2a and $\mathrm{b}$ show the estimated CMV prevalence (solid lines) in females and males as a function of age. At the age of 6 months, about 17.2\% (95\%CrI 14.7-20.0\%) of females and males were estimated to be seropositive. The prevalence of seronegative persons decreased gradually with age to $33.7 \%(95 \% \mathrm{CrI} 31.2-36.2 \%)$ and $36.5 \%$ $(95 \%$ CrI $33.9-38.8 \%)$ at 80 years in females and males, respectively. The estimated prevalence of persons with low antibody concentrations varied from 16.6 to $29.0 \%$ in females and from 16.6 to $33.6 \%$ in males. The prevalence of persons with high antibody concentrations gradually increased with age, reaching $48.4 \%$ (95\% CrI 43.7-53.7\%) at 80 years in females and $34.7 \%$ (95\%CrI $29.7-41.0 \%)$ in males.

The estimated reactivation rates ranged from 0.031 to 0.058 per year for females and from 0.018 to 0.038 per year for males (Fig. 2c). For all ages, the reactivation rate was higher in females than in males. The estimated probabilities of vertical transmission and of cCMV from acutely infected pregnant women were 0.37 (95\% CrI 0.31-0.43) and 0.17 (95\% CrI 0.11-0.26), respectively. Figure S3 gives an overview of all parameter estimates. In the absence of interventions, the estimated basic reproduction number was 1.30 (95\%CrI 1.15-1.43). The analyses show that without vertical transmission the basic reproduction number would be 0.95 (95\%CrI 0.80-1.12). Combining the incidence estimates of the transmission model with an estimate of 3.034 DALYs per case of CCMV yielded an estimated burden of cCMV in the Netherlands of 3527 DALYs per year (95\%CrI 1397-7098) or 20.75 DALYs per 100,000 population per year $(95 \% \mathrm{CrI} 8.22-41.75)$ (see Additional file 1: Appendix).

\section{Vaccination dynamics}

All interventions are able to reduce the incidence arising through each route of transmission. The quantitative impact, however, depends strongly on the intervention (hygiene measures, vaccination during pregnancy, universal vaccination), mode of action of the vaccine (prevention of infection vs prevention of (re-)infection and reactivation), vaccination age and sex, and duration of protection by vaccination. Figure 3 shows results for vaccination of women, with a vaccine that protects for 10 years against infection and re-infection or reactivation. Here, the age at vaccination is varied from 10 to 25 years, and to 50 years, while the effectively vaccinated proportion is $30 \%, 70 \%$, or $100 \%$. Figure 3 a shows that for vaccination of 

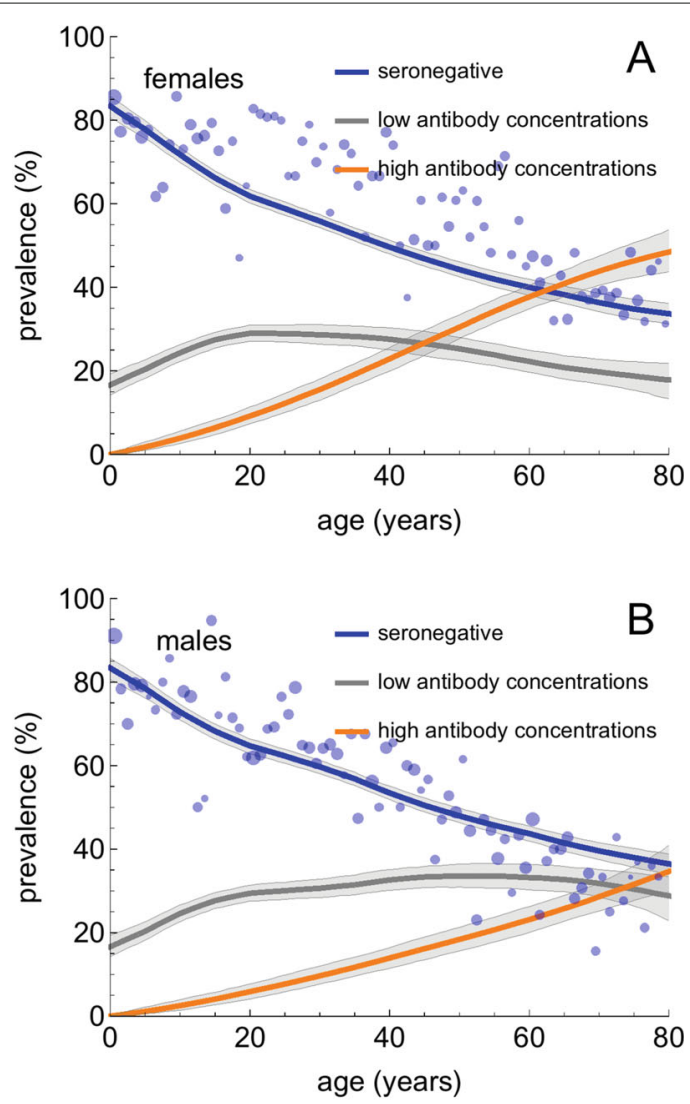

0.14

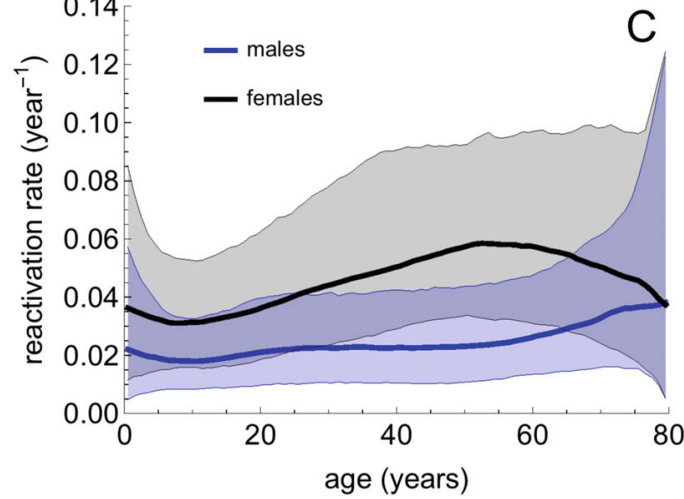

Fig. 2 Overview of the estimation results. $\mathbf{a}, \mathbf{b}$ The estimated age-specific prevalence of seronegative persons, seropositive persons with low antibody concentrations, and seropositive persons with high antibody concentrations in females (a) and males (b), respectively. c The age-specific reactivation rates per year for females (black) and males (blue), respectively. The solid lines represent the estimated medians, and the shaded regions correspond to 95\% credible intervals obtained from 1000 parameter samples from the posterior distribution. The circles are seroprevalence data [33] indicating the fraction of samples that would be classified as seronegative with the cut-off specified by the supplier of the assay. The circle size gives a measure for the number of samples in 1-year age groups. The number of samples per 1-year age group is approximately 35 (females) and 30 (males). Note that the seroprevalence is estimated with high precision and that credible intervals for the reactivation rates are broad
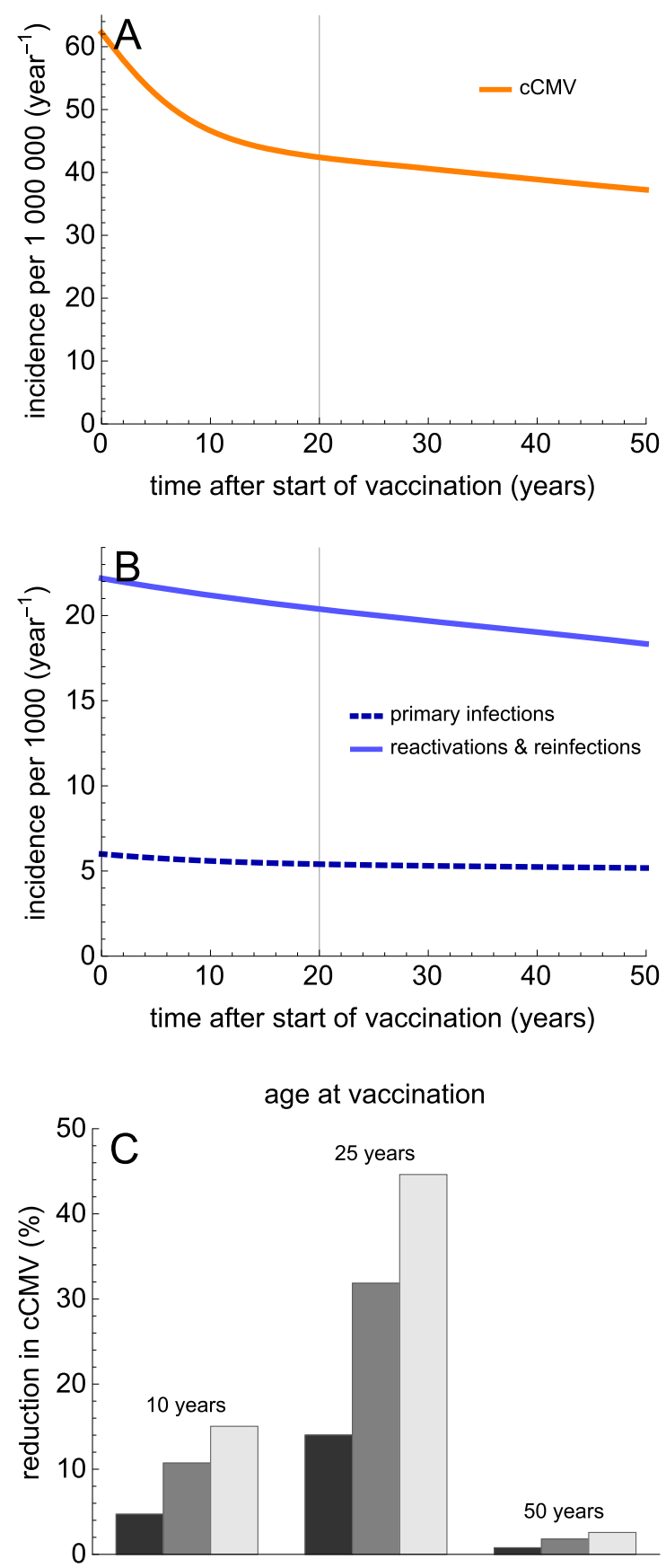

proportion of effectively vaccinated women (\%)

$$
30 \square 70 \square 100
$$

Fig. 3 Universal vaccination of females with a vaccine preventing (re-)infection and reactivation. a The incidence of CCMV. $\mathbf{b}$ The incidence of primary infections and re-infections/reactivations during the first 50 years after the start of vaccination of 25-year-old women. The proportion of effectively vaccinated women (vaccination coverage $\times$ vaccine efficacy) is $70 \%$, and the duration of protection is 10 years. $\mathbf{c}$ The reduction in CCMV after 20 years for different vaccination ages (10,25, and 50 years) and proportions of effectively vaccinated women 
25-year-old women the incidence of cCMV decreases quite rapidly during the first $10-20$ years after the start of the vaccination program, from 62 cases per million per year at the start to 42 cases per million per year after 20 years. After this, the incidence of cCMV keeps decreasing due to the indirect benefits of vaccination, albeit at a much slower pace (1 case per million per 5 years). In this scenario, the incidence of primary infection and re-infection/reactivation also decrease, but at a relatively slow pace (Fig. $3 \mathrm{~b}$ and Table 1).

Figure $3 \mathrm{c}$ illustrates that the reduction in cCMV after 20 years also depends strongly on the age at vaccination and proportion of the population that is effectively protected after vaccination. Unsurprisingly, the reduction in $\mathrm{CCMV}$ increases with increasing vaccinated proportion. Further, reductions in cCMV are generally small for vaccination programs in women who are past their reproductive age (e.g., 50 years), because there are no direct effects of vaccination on cCMV and only limited indirect effects on cCMV via reduction of prevalence in the population. The highest benefits are obtained if vaccination is given at an age where vaccine protection covers the main reproductive period (i.e., 25 years). Then, there are substantial benefits, to the extent that more than $30 \%$ and $40 \%$ of cCMV can be prevented if $70 \%$ and $100 \%$ of women are effectively vaccinated, respectively. Interestingly, where vaccination of women at 25 years is more effective than vaccination at 10 years in reducing cCMV (after 20 years), the latter strategy is slightly more effective at reducing primary infections (Table $1 ; 10.5 \%$ vs $9.9 \%$ ).

\section{A comparison of scenarios}

Table 1 shows an overview of the impact of interventions on the reduction in the incidence of cCMV (henceforth called birth prevalence) and incidence of primary infection and re-infection/reactivation within 20 years. Intervention strategies aimed at reducing transmission from mother to child can yield substantial reductions of cCMV. Universal vaccination of females at the age of 10 and 25 years with a vaccine preventing (re-)infection and reactivation leads to $10.8 \%(95 \% \mathrm{CrI} 9.7-12.8 \%)$ and $31.7 \%$ (95\% CrI 30.6-33.6\%) reduction of cCMV, respectively. If the vaccine administered to females prevents infection only, the reduction of cCMV would be $5.3 \%$ (95\%CrI $4.2-6.0 \%)$ and $8.4 \%$ (95\%CrI 6.6-10.7\%). Vaccination of males and females at the age of 6 months, to the contrary, leads to smaller reduction in cCMV (median of 5.4\%) but a much larger reduction in primary infections (median of 18.7\%). Vaccination during pregnancy is very effective in reducing cCMV (median of $71.4 \%$ ), but it has the least impact on primary infections. Hygiene measures are ineffective in reducing any type of infections.
If $70 \%$ of persons are effectively vaccinated and the duration of protection is 10 years, the effective reproduction numbers for all scenarios are above 1 (Table 1). This means that CMV elimination cannot be achieved. We further explored for which proportion of effectively vaccinated persons and the duration of protection CMV elimination would be feasible. Figure 4 shows the regions of persistence and elimination for the universal vaccination with a vaccine protecting against (re-)infection and reactivation. The black circle indicates parameter values used in Table 1. Our analyses show that for all vaccination strategies, the longer the duration of protection, the smaller the proportion of persons that needs to be effectively vaccinated to eliminate CMV. Vaccinated proportions and durations of protection required to achieve elimination by vaccinating males and females at the age of 6 months and by the same strategy at the age of 10 years are similar (e.g., $60 \%$ and 20 years, correspondingly). However, vaccinating effectively the same proportion of females only at the age of 10 or 25 years would require a vaccine with a longer duration of protection for elimination (i.e., 27 years for $60 \%)$.

\section{Discussion}

Congenital CMV is a disease with serious and lifelong sequelae $[3,42]$. We estimated that the burden of cCMV in the Netherlands is around 3527 DALYs per year $(95 \% \mathrm{CrI}$ : 1397-7098) or 20.75 DALYs per 100,000 persons per year (95\%CrI 8.22-41.75). This burden is quite high and comparable to the burden of pertussis (3235 DALYs per year), chlamydia (3551 DALYs per year), or campylobacter (3314 DALYs per year), against which vaccination programs or other types of interventions are in place in the Netherlands [50]. Worldwide, the burden of cCMV is estimated to be even higher, as maternal prevalence of infection is higher in developing countries than in Europe and North America. Here, we have shown that several vaccination strategies have the potential to reduce the birth prevalence of cCMV in a time span of 20 years, thereby significantly reducing disease burden as measured by DALYs prevented (Table 1). Of these, vaccination during pregnancy is expected to be the most effective in preventing cCMV and its related disease burden as it specifically targets those at risk. Vaccination during pregnancy is expected to prevent almost 50,000 DALYs over a time period of 20 years, which is about $70 \%$ of all DALYs that would occur without intervention. After 20 years of vaccination, the annual disease burden from $\mathrm{CCMV}$ would be reduced to around 1000 DALYs per year or 6 DALYs per 100,000 population per year, which would be lower than the burden from gonorrhea (1271 DALYs per year) or invasive meningococcal disease (1065 DALYs per year) [50]. Altogether, our modeling and burden estimates before and 
Table 1 Impact of interventions on cCMV, primary infection, and re-infection/reactivation

\begin{tabular}{|c|c|c|c|c|c|}
\hline \multirow{5}{*}{ Intervention scenario } & \multirow{5}{*}{$\begin{array}{l}\text { Reduction \% } \\
\text { birth prevalence } \\
\text { cCMV } \\
\text { median }(95 \% \mathrm{Crl})\end{array}$} & \multirow{5}{*}{$\begin{array}{l}\text { Reduction \% } \\
\text { incidence } \\
\text { primary } \\
\text { infection } \\
\text { median }(95 \% \mathrm{Crl})\end{array}$} & \multicolumn{3}{|l|}{ Reduction \% } \\
\hline & & & incidence & Effective & \\
\hline & & & re-infection/ & reproduction & DALYS \\
\hline & & & reactivation & number & prevented \\
\hline & & & median $(95 \% \mathrm{Crl})$ & median (95\% Crl) & median $(95 \% \mathrm{Crl})$ \\
\hline \multicolumn{6}{|l|}{ Universal vaccination } \\
\hline \multicolumn{6}{|c|}{ Prevention of (re-)infection and reactivation } \\
\hline 6-month-old boys and girls & $5.4(4.1-7.7)$ & $18.7(16.6-21.9)$ & $5.2(3.9-8.0)$ & $1.09(0.98-1.20)$ & $1,446(1,007-2,304)$ \\
\hline 10-year-old boys and girls & $11.7(10.2-14.1)$ & $17.2(15.2-20.3)$ & $7.0(5.0-10.0)$ & $1.10(0.98-1.21)$ & $3,558(2,731-4,979)$ \\
\hline 10-year-old girls & $10.8(9.7-12.8)$ & $10.5(9.1-13.2)$ & $4.7(3.5-7.2)$ & $1.13(1.01-1.26)$ & $3,267(2,515-4,386)$ \\
\hline 25-year-old women & $31.7(30.6-33.6)$ & $9.9(8.6-12.5)$ & $7.9(6.5-10.8)$ & $1.11(0.98-1.22)$ & $15,969(12,560-19,993)$ \\
\hline \multicolumn{6}{|l|}{ Prevention of infection } \\
\hline 6-month-old boys and girls & $3.2(2.3-4.4)$ & $15.4(13.9-17.7)$ & $2.4(1.8-3.9)$ & $1.13(1.01-1.24)$ & $788(465-1,091)$ \\
\hline 10-year-old boys and girls & $5.6(4.4-6.7)$ & $12.5(11.0-14.3)$ & $2.2(1.5-3.3)$ & $1.16(1.04-1.28)$ & $1,584(1,063-2,087)$ \\
\hline 10-year-old girls & $5.3(4.2-6.0)$ & $7.0(6.2-8.1)$ & $1.5(1.0-2.3)$ & $1.19(1.07-1.31)$ & $1,500(1,012-1,918)$ \\
\hline 25-year-old women & $8.4(6.6-10.7)$ & $4.5(4.1-4.9)$ & $1.1(0.9-1.5)$ & $1.25(1.12-1.36)$ & $4,227(2,646-4,968)$ \\
\hline Vaccination during pregnancy & 71.4 (71.0-71.9) & $2.4(0.3-4.8)$ & $5.9(4.3-8.5)$ & $1.05(0.92-1.18)$ & $49,705(40,280-61,435)$ \\
\hline Hygienic measures & $1.8(1.0-2.8)$ & $3.0(2.5-4.0)$ & $0.9(0.6-1.7)$ & $1.27(1.13-1.38)$ & $819(470-1278)$ \\
\hline
\end{tabular}

The reductions are evaluated 20 years after the start of the intervention. The proportion of effectively vaccinated persons (vaccination coverage $\times$ vaccine efficacy) is $70 \%$, and the average duration of protection is 10 years. Hygiene measures are modeled as a $70 \%$ reduction in infectious contacts between women of reproductive age (15-50 years) and young children ( $0-5$ years). The effective reproduction number is defined as the average number of secondary infections at the start of an epidemic with one infected individual introduced in a population where $70 \%$ of persons are effectively vaccinated. This number smaller than 1 indicates that a given intervention is going to lead to the disease elimination in the long run. The burden of disease prevented by an intervention over the time period of 20 years in the Dutch population is given by the number of DALYs prevented

after vaccination indicate that maternal vaccination with an effective vaccine can substantially reduce the burden of cCMV.

An alternative would be indiscriminate vaccination of women with a vaccine preventing (re-)infection and reactivation before the age at which they generally will have children (20-35 years in the Netherlands). However, in terms of burden prevented, this strategy is much less effective than vaccination of pregnant women with approximately 20,000 DALYs or $30 \%$ of the expected burden prevented over a time period of 20 years. On the other hand, a general vaccination program targeting young women has the advantage that it not only reduces cCMV in the short run (albeit less so than vaccination during pregnancy), but has a larger contribution to reducing CMV circulation in the population. This potentially has as a byproduct some added advantages, for instance by indirectly protecting solid organ and stem cell transplants, and other persons with reduced immunocompetence. In our analyses, both vaccination strategies could in the long run even lead to elimination, but this would require a sufficiently high vaccination coverage together with a long duration of protection by vaccination spanning the reproductive life span (e.g., $80 \%$ of women effectively vaccinated and 15 years of protection; Fig. 4). However, since CMV causes a lifelong infection and onward transmission is possible during reactivation, the timescale on which elimination could be achieved is very long, in the order of more than 100 years.

In our analyses, all other strategies including pediatric vaccination only prevent small proportions of the expected disease burden in the relevant short term (20 years, say). In fact, in the short term, a pediatric vaccination program is expected to be the least effective vaccination strategy in reducing cCMV but most effective at reducing primary infections. In addition, pediatric vaccination also can achieve elimination with lower effort (e.g., lower vaccinated proportion or shorter duration of protection) when compared to vaccination of young women and could be a better vaccination strategy if the goal is to reduce overall CMV transmission in the long term. This is due to the fact that the indirect effects of vaccination on horizontal transmission are strongest when the vaccine is administered at an age when contact rates are highest (i.e., children). Combining universal childhood vaccination and vaccination of pregnant women would thus seem the optimal strategy. We hope that our analyses will help to support future health economic assessments of potential CMV vaccine candidates, although our model may require modifications to support additional strategies 


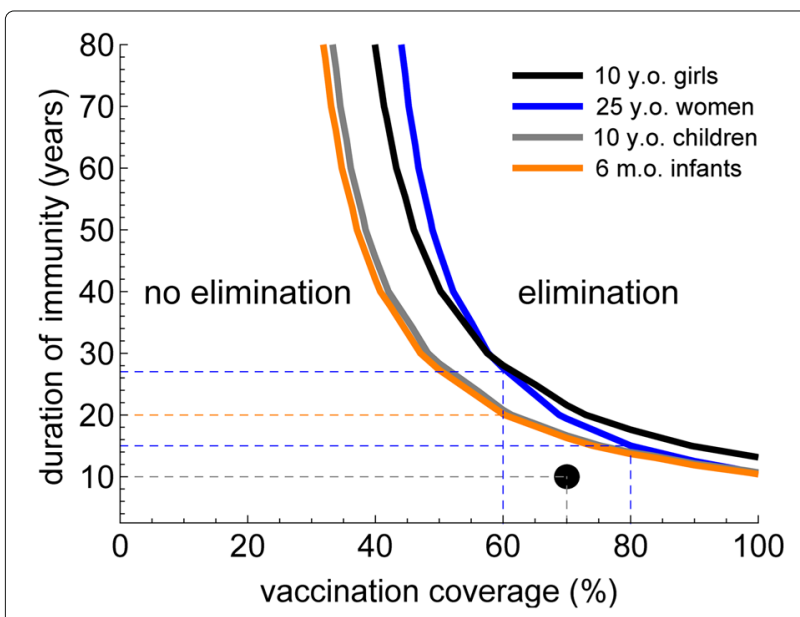

Fig. 4 Elimination and persistence for universal vaccination with a vaccine preventing (re-)infection and reactivation. CMV elimination is feasible for effectively vaccinated proportions and durations of protection above the curve, while below the curve CMV cannot be eliminated. The curves correspond to the effective reproduction number equal to 1 . The parameter values used in Table 1 are depicted as the black circle (70\% and 10 years). For all vaccination strategies, the longer the duration of protection, the smaller proportion of persons would need to be effectively vaccinated to eliminate CMV. Vaccination of females only at the age of 10 or 25 years would require a vaccine with a longer duration of protection for elimination than vaccination of both males and females at the age of 6 months or at 10 years (e.g., about 27 years vs 20 years if $60 \%$ of the target group are effectively vaccinated)

(e.g., for other populations such as high-risk groups) and the combination of strategies (e.g., universal childhood vaccination and vaccination of pregnant women) that we did not consider here.

The feasibility of the different vaccination scenarios deserves special attention. In the comparative analyses (Table 1), we assumed that the proportion of persons that is effectively vaccinated is $70 \%$ for all scenarios. This proportion is high but could potentially be achieved for pregnant women, infants, or adolescent children if CMV vaccination is implemented in immunization programs for other diseases. For adult vaccination, much lower coverage could be expected, and the effects on cCMV will be respectively smaller. For example, if only $40 \%$ of 25 -year-old women are effectively vaccinated with a vaccine preventing (re-)infection and reactivation, the birth prevalence of cCMV is reduced by $18.5 \%$, as compared to $31.7 \%$ when the proportion of effectively vaccinated women is $70 \%$ (Table 1 ).

Maternal acquisition of CMV via contact with a child under 3 years of age is an important transmission route for pregnant women [25]. Currently recommended hygienic measures for reducing the risk of CMV acquisition during pregnancy center around minimizing contact with saliva and urine from young children by not sharing food, utensils, or cups with a child and also washing hands after changing diapers. The effect of hygiene intervention on CMV primary infection during pregnancy was demonstrated in controlled studies [25]. Our model, however, predicts that hygiene measures aimed to protect women of reproductive age with exposure to young children would have little impact on reducing cCMV. Decreasing infectious contacts between women and young children by $70 \%$ results only in $1.8 \%$ reduction of cCMV, because women continue to acquire CMV via contacts with older persons (e.g., older children or partners). This suggests that hygiene counseling for pregnant women should not be focused on prevention of potential infectious contacts with young children but rather with persons of all ages. In fact, in studies demonstrating the effectiveness of hygiene intervention $[24,25]$ women might have used hygiene precautions with a broader range of contacts, in particular, with their (seropositive) spouses who, in some studies, received detailed hygiene information as well.

Main strengths of our study are that all known transmission routes are included in one transmission model analysis and that parameters of the model have been estimated using two large unbiased population studies $[33,34]$. Thus, the baseline for the analyses is consistent with the epidemiology of CMV. By evaluating a suite of vaccination scenarios, our analyses have provided, for the first time, a sound empirical basis for comparative analysis of possible interventions, among which hygiene measures and vaccination during pregnancy were considered for the first time. This comparison suggests that vaccination of pregnant women will result in a greater decline in cCMV than vaccination of infants and/or young women considered in previous modeling studies [38-40], therefore highlighting a need for the development of a vaccine that can be administered during pregnancy. Although our model was parameterized using data from the Netherlands, conclusions can be drawn also for the possible impact of vaccination in resource-limited settings, where maternal prevalence is higher. Our analysis suggests that vaccination during pregnancy could have an even higher impact on disease burden in these settings, and also hygiene measures could potentially be more effective. Vaccination and counseling could be integrated in antenatal health care, which reaches women in reproductive age in many resource-poor settings.

A number of limitations need scrutiny. First, we have restricted our statistical analyses to persons of western ethnicity. This was done to not overcomplicate an already complex transmission model. However, it is known that CMV prevalence in the Netherlands is higher in persons of non-western ethnicity [33], and how this would impact the effectiveness of vaccination is unknown. Second, estimates of the reactivation rates, infectivity of re-infection 
and reactivation, and probability to move from the class with low to the class with high antibody concentrations depend sensitively on the assumed prior distributions of the reactivation rates. The sensitivity analyses for prior distributions for these rates demonstrate that the impact of vaccination, in particular the estimated reductions in cCMV, primary infections, re-infections, and reactivations are quantitatively very similar to the results in the main text (Table S2). Hence, while considerable uncertainty surrounds the actual magnitude of the reactivation rates and associated parameters, the impact on the effectiveness of vaccination is found to be small.

\section{Conclusions}

In conclusion, our study has provided a comparative analysis of the expected effectiveness of vaccination against CMV and has shown that several options exist to reduce the incidence of congenital CMV in a time span of 20 years. Depending on the proportion of the target group that is effectively vaccinated and the duration of protection after vaccination, significant long-term reductions in the overall circulation of CMV at the population level also seem within reach. It is even conceivable that a vaccination campaign with an effective vaccine and high vaccination coverage ( $>80 \%$ ) could in the long run lead to elimination. In view of the associations of CMV infection with accelerated aging of the immune system $[8,51,52]$, such long-term benefits could in the long run factor in heavily in future analyses of the effectiveness and cost-effectiveness of CMV vaccination.

\section{Supplementary information}

Supplementary information accompanies this paper at

https://doi.org/10.1186/s12916-020-01629-3.

Additional file 1: Appendix: This appendix contains all mathematical details of the analyses presented in the main text.

\section{Abbreviations}

CCMV: Congenital cytomegalovirus infection; CMV: Cytomegalovirus; DALYs: Disability-adjusted life years

\section{Acknowledgements}

Christiaan van Dorp (Los Alamos National Laboratory, New Mexico, USA) Sophia de Jong (Free University Amsterdam, The Netherlands), and Odo Diekmann (Mathematical Institute, Utrecht University, The Netherlands) are gratefully acknowledged for the discussion and help in the early stages of this research. We thank Brecht Devleesschauwer (Scientific Institute of Public Health, Brussels, Belgium) for the advice on the DALY computation.

\section{Authors' contributions}

GR, MEK, and MvB developed the conceptual framework of the study. GR developed the model and performed the model analyses. MvB performed the data analyses. MEK performed the DALY computation. GR and MvB wrote the manuscript. MEK, FvdK, DvB, MK, and ACV contributed to the interpretation of the results and provided critical review of the manuscript. All authors approved its final version.

\section{Funding}

This study was supported by RIVM's Strategic Programme (project S/113007, awarded to MvB) and the EU IMI project on Vaccines and Infectious Diseases in the Ageing Population project (VITAL, awarded to DVB). VITAL has received funding from the Innovative Medicines Initiative 2 Joint Undertaking under grant agreement no. 806776

\section{Availability of data and materials}

The datasets generated and/or analyzed during the current study are available in the GitHub repository, https://github.com/mvboven/cmv-vaccination.

\section{Ethics approval and consent to participate}

The serological study (Pienter2) was approved by the Medical Ethics Testing Committee of the foundation of therapeutic evaluation of medicines (METC-STEG) in Almere, the Netherlands (clinical trial number: ISRCTN 20164309). All participants or their legal representatives had given written informed consent. The retrospective birth cohort study (CROCUS) was approved by the ethical committee of the Leiden University Medical Center in Leiden and is registered in the Dutch Trial Register under NTR3582.

\section{Consent for publication}

Not applicable.

\section{Competing interests}

The authors declare that they have no competing interests.

\section{Author details}

1 Julius Center for Health Sciences and Primary Care, University Medical Center Utrecht, Utrecht University, Utrecht, The Netherlands. ${ }^{2}$ Center for Infectious Disease Control, National Institute for Public Health and the Environment, Bilthoven, The Netherlands. ${ }^{3}$ Department of Medical Microbiology, Leiden University Medical Center, Leiden, The Netherlands. ${ }^{4}$ BiolSI - Biosystems \& Integrative Sciences Institute, Faculdade de Ciências, Universidade de Lisboa, Lisboa, Portugal. ${ }^{5}$ Center for Translational Immunology, University Medical Center Utrecht, Utrecht University, Utrecht, The Netherlands.

Received: 9 February 2020 Accepted: 13 May 2020

Published online: 02 July 2020

\section{References}

1. Cannon MJ, Schmid DS, Hyde TB. Review of cytomegalovirus seroprevalence and demographic characteristics associated with infection. Rev Med Virol. 2010;20(4):202-13.

2. Dollard SC, Grosse SD, Ross DS. New estimates of the prevalence of neurological and sensory sequelae and mortality associated with congenital cytomegalovirus infection. Rev Med Virol. 2007;17(5):355-63.

3. Manicklal S, Emery VC, Lazzarotto T, Boppana SB, Gupta RK. The "silent" global burden of congenital cytomegalovirus. Clin Microbiol Rev. 2013;26(1):86-102.

4. Ramanan P, Razonable RR. Cytomegalovirus infections in solid organ transplantation: a review. Infect Chemother. 2013;45(3):260-71.

5. Styczynski J. Who is the patient at risk of CMV recurrence: a review of the current scientific evidence with a focus on hematopoietic cell transplantation. Infect Dis Ther. 2018;7(1):1-16.

6. Gkrania-Klotsas E, Langenberg C, Sharp SJ, Luben R, Khaw KT, Wareham NJ. Higher immunoglobulin $\mathrm{G}$ antibody levels against cytomegalovirus are associated with incident ischemic heart disease in the population-based EPIC-Norfolk cohort. J Infect Dis. 2012;206(12):1897-903.

7. Pawelec G. Immunosenenescence: role of cytomegalovirus. Exp Gerontol. 2014;54:1-5.

8. Klenerman P, Oxenius A. T cell responses to cytomegalovirus. Nat Rev Immunol. 2016;16(6):367-77.

9. Merani S, Pawelec G, Kuchel GA, McElhaney JE. Impact of aging and cytomegalovirus on immunological response to influenza vaccination and infection. Front Immunol. 2017:8:784

10. Griffiths PD. Burden of disease associated with human cytomegalovirus and prospects for elimination by universal immunisation. Lancet Infect Dis. 2012;12:790-98.

11. Lanzieri TM, Dollard SC, Bialek SR, Grosse SD. Systematic review of the birth prevalence of congenital cytomegalovirus infection in developing 
countries. Int J Infect Dis. 2014;22:44-8. https://doi.org/10.1016/j.ijid.2013. 12.010.

12. Ludwig A HH. Epidemiological impact and disease burden of congenital cytomegalovirus infection in Europe. Euro Surveill. 2009;14(9):1-7.

13. Smit GSA, Padalko E, Van Acker J, Hens N, Dorny P, Speybroeck N, Devleesschauwer B. Public health impact of congenital toxoplasmosis and cytomegalovirus infection in Belgium, 2013: a systematic review and data synthesis. Clin Infect Dis. 2017;65(4):661-8. http://oup.prod.sis.lan/ cid/article-pdf/65/4/661/19378691/cix344.pdf https://doi.org/10.1093/ $\mathrm{cid} / \mathrm{cix344.}$

14. Morton C, Nance W. Newborn hearing screening - a silent revolution. N Engl J Medl. 2006;354:2151-64.

15. Staras SA, Dollard SC, Radford KW, Flanders WD, Pass RF, Cannon MJ. Seroprevalence of cytomegalovirus infection in the United States, 1988-1994. Clin Infect Dis. 2006;43(9):1143-51.

16. Staras SA, Flanders WD, Dollard SC, Pass RF, McGowan JE, Cannon MJ. Cytomegalovirus seroprevalence and childhood sources of infection: a population-based study among pre-adolescents in the United States. J Clin Virol. 2008;43(3):266-71.

17. Bate SL, Dollard SC, Cannon MJ. Cytomegalovirus seroprevalence in the United States: the national health and nutrition examination surveys, 1988-2004. Clin Infect Dis. 2010;50(11):1439-47.

18. Griffiths P, Plotkin S, Mocarski E, Pass R, Schleiss M, Krause P, Bialek S. Desirability and feasibility of a vaccine against cytomegalovirus. Vaccine. 2013;31 Suppl 2:197-203.

19. Bernstein DI. Congenital cytomegalovirus: a "now" problem-no really, now. Clin Vaccine Immunol. 2017;24(1):e00491-16. https://doi.org/10. 1128/CVI.00491-16.

20. Schleiss MR, Permar SR, Plotkin SA. Progress toward development of a vaccine against congenital cytomegalovirus infection. Clin Vaccine Immunol. 2017;24(12):. https://doi.org/10.1128/CVI.00268-17 https://cvi. asm.org/content/24/12/e00268-17.full.pdf.

21. Bialas KM, Permar SR. The march towards a vaccine for congenital CMV: rationale and models. PLoS Pathog. 2016;12(2):1005355.

22. Plotkin SA, Boppana SB. Vaccination against the human cytomegalovirus. Vaccine. 2018. https://doi.org/10.1016/j.vaccine.2018.02.089.

23. Adler S, Finney J, Manganello A, Best A. Prevention of child-to-mother transmission of cytomegalovirus by changing behaviors: a randomized controlled trial. Pediatr Infect Dis J. 1996;15(3):240-6. https://doi.org/10. 1097/00006454-199603000-00013

24. Vauloup-Fellous C, Picone O, Cordier A-G, Parent-du-Châtelet I, Senat M-V, Frydman R, Grangeot-Keros L. Does hygiene counseling have an impact on the rate of CMV primary infection during pregnancy?: results of a 3-year prospective study in a french hospital. J Clin Virol. 2009;46:49-53. https://doi.org/10.1016/j.jcv.2009.09.003.

25. Adler SP, Nigro G. Prevention of maternal-fetal transmission of cytomegalovirus. Cli Infect Dis. 2013;57(Suppl 4):189-92. https://doi.org/ 10.1093/cid/cit585. http://oup.prod.sis.lan/cid/article-pdf/57/suppl_4/ S189/20911485/cit585.pdf.

26. Revello MG, Tibaldi C, Masuelli G, Frisina V, Sacchi A, Furione M, Arossa A, Spinillo A, Klersy C, Ceccarelli M, Gerna G, Todros T. Prevention of primary cytomegalovirus infection in pregnancy. EBioMedicine. 2015;2(9): 1205-10. https://doi.org/10.1016/j.ebiom.2015.08.003.

27. Cannon MJ, Hyde TB, Schmid DS. Review of cytomegalovirus shedding in bodily fluids and relevance to congenital cytomegalovirus infection. Rev Med Virol. 2011;21(4):240-55.

28. Kenneson A, Cannon MJ. Review and meta-analysis of the epidemiology of congenital cytomegalovirus (CMV) infection. Rev Med Virol. 2007;17(4): 253-76.

29. Pass RF, Anderson B. Mother-to-child transmission of cytomegalovirus and prevention of congenital infection. J Pediatric Infect Dis Soc. 2014;3 Suppl 1:2-6.

30. Hamprecht K, Maschmann J, Vochem M, Dietz K, Speer CP, Jahn G. Epidemiology of transmission of cytomegalovirus from mother to preterm infant by breastfeeding. Lancet. 2001;357(9255):513-8.

31. Prendergast AJ, Goga AE, Waitt C, Gessain A, Taylor GP, Rollins N, Abrams EJ, Lyall EH, de Perre PV. Transmission of CMV, HTLV-1, and HIV through breastmilk. Lancet Child Adolesc Health. 2019;3(4):264-73.

32. Boppana SB, Fowler KB. Persistence in the population: epidemiology and transmisson. In: Arvin A, Campadelli-Fiume G, Mocarski E, Moore PS,
Roizman B, Whitley R, Yamanishi K, editors. Human herpesviruses: biology, therapy, and immunoprophylaxis. Cambridge: Cambridge University Press; 2007.

33. Korndewal MJ, Mollema L, Tcherniaeva I, van der Klis F, Kroes ACM, Oudesluys-Murphy AM, Vossen ACTM, de Melker HE. Cytomegalovirus infection in the Netherlands: seroprevalence, risk factors, and implications. J Clin Virol. 2015;63:53-58. https://doi.org/10.1016/j.jcv.2014.11.033.

34. Korndewal MJ, Vossen ACTM, Cremer J, van Binnendijk RS, Kroes ACM, van der Sande MAB, Oudesluys-Murphy AM, de Melker HE. Disease burden of congenital cytomegalovirus infection at school entry age: study design, participation rate and birth prevalence. Epidemiol Infect. 2016;144(7):1520-7. https://doi.org/10.1017/S0950268815002708.

35. Dempsey AF, Pangborn HM, Prosser LA. Cost-effectiveness of routine vaccination of adolescent females against cytomegalovirus. Vaccine. 2012;30(27):4060-6. https://doi.org/10.1016/j.vaccine.2012.04.011.

36. N'Diaye DS, Launay O, Picone O, Tsatsaris V, Azria E, Rozenberg F, Schwarzinger M, Yazdanpanah Y. Cost-effectiveness of vaccination against cytomegalovirus (CMV) in adolescent girls to prevent infections in pregnant women living in France. Vaccine. 2018;36(10):1285-96. https:// doi.org/10.1016/j.vaccine.2018.01.042.

37. Griffiths PD, McLean A, Emery VC. Encouraging prospects for immunisation against primary cytomegalovirus infection. Vaccine. 2001;19(11):1356-62. https://doi.org/10.1016/50264-410X(00)00377-7.

38. Lanzieri TM, Bialek SR, Ortega-Sanchez IR, Gambhir M. Modeling the potential impact of vaccination on the epidemiology of congenital cytomegalovirus infection. Vaccine. 2014;32(30):3780-6. https://doi.org/ 10.1016/j.vaccine.2014.05.014.

39. Hogea C, Dieussaert I, Effelterre TV, Guignard A, Mols J. A dynamic transmission model with age-dependent infectiousness and reactivation for cytomegalovirus in the United States: potential impact of vaccination strategies on congenital infection. Hum Vaccines Immunotherapeutics. 2015;11(7):1788-802. https://doi.org/10.1080/21645515.2015.1016665. PMID: 25984886.

40. Alfaro-Murillo JA, Townsend JP, Galvani AP. Optimizing age of cytomegalovirus screening and vaccination to avert congenital disease in the US. Vaccine. 2016;34(2):225-9. https://doi.org/10.1016/j.vaccine.2015. 11.039 .

41. van Boven M, van de Kassteele J, Korndewal MJ, van Dorp CH, Kretzschmar M, van der Klis F, de Melker HE, Vossen AC, van Baarle D. Infectious reactivation of cytomegalovirus explaining age- and sex-specific patterns of seroprevalence. PLoS Comput Biol. 2017;13(9): 1-18. https://doi.org/10.1371/journal.pcbi.1005719.

42. Korndewal MJ, Oudesluys-Murphy AM, Kroes ACM, van der Sande MAB, de Melker HE, Vossen ACTM. Long-term impairment attributable to congenital cytomegalovirus infection: a retrospective cohort study. 1261-1268. 59(12):. https://doi.org/10.1111/dmcn.13556.

43. van der Klis FR, Mollema L, Berbers GA, de Melker HE, Coutinho RA. Second national serum bank for population-based seroprevalence studies in the Netherlands. Neth J Med. 2009;67:301-8.

44. van de Kassteele J, van Eijkeren J, Wallinga J. Efficient estimation of age-specific social contact rates between men and women. Ann Appl Stat. 2017:11:320-39.

45. Diekmann O, Heesterbeek H, Britton T. Mathematical tools for understanding infectious disease dynamics. Princeton, NJ: Princeton University Press; 2013.

46. Carpenter B, Gelman A, Hoffman M, Lee D, Goodrich B, Betancourt M, Brubaker M, Guo J, Li P, Riddell A. Stan: a probabilistic programming language. J Stat Softw Artic. 2017;76(1):1-32. https://doi.org/10.18637/jss. v076.i01.

47. Krause PR, Bialek SR, Boppana SB, Griffiths PD, Laughlin CA, Ljungman P, Mocarski ES, Pass RF, Read JS, Schleiss MR, Plotkin SA. Priorities for CMV vaccine development. Vaccine. 2013;32(1):4-10. https://doi.org/10 1016/j.vaccine.2013.09.042.

48. van den Driessche P, Watmough J. Reproduction numbers and sub-threshold endemic equilibria for compartmental models of disease transmission. Math Biosci. 2002;180(1):29-48. https://doi.org/10.1016/ S0025-5564(02)00108-6.

49. Diekmann O, Heesterbeek JAP, Roberts MG. The construction of next-generation matrices for compartmental epidemic models. J R Soc Interface. 2010;7(47):873-85. https://doi.org/10.1098/rsif.2009.0386 http://arxiv.org/abs/https://royalsocietypublishing.org/doi/pdf/10.1098/ rsif.2009.0386. 
50. van Lier A, McDonald SA, Bouwknegt M, group E, Kretzschmar ME, Havelaar AH, Mangen M-JJ, Wallinga J, de Melker HE. Disease burden of 32 infectious diseases in the Netherlands, 2007-2011. PLOS ONE. 2016;11(4):1-25. https://doi.org/10.1371/journal.pone.0153106.

51. Sansoni P, Vescovini R, Fagnoni FF, Akbar A, Arens R, Chiu YL, Ci?in-?ain L, Dechanet-Merville J, Derhovanessian E, Ferrando-Martinez S, Franceschi C, Frasca D, Fulop T, Furman D, Gkrania-Klotsas E, Goodrum F, Grubeck-Loebenstein B, Hurme M, Kern F, Lilleri D, Lopez-Botet M, Maier AB, Marandu T, Marchant A, Mathei C, Moss P, Muntasell A, Remmerswaal EB, Riddell NE, Rothe K, Sauce D, Shin EC, Simanek AM, Smithey MJ, Soderberg-Naucler C, Solana R, Thomas PG, van Lier R, Pawelec G, Nikolich-Zugich J. New advances in CMV and immunosenescence. Exp Gerontol. 2014;55:54-62.

52. de Bourcy CF, Angel CJ, Vollmers C, Dekker CL, Davis MM, Quake SR. Phylogenetic analysis of the human antibody repertoire reveals quantitative signatures of immune senescence and aging. Proc Natl Acad Sci U S A. 2017;114(5):1105-10.

\section{Publisher's Note}

Springer Nature remains neutral with regard to jurisdictional claims in published maps and institutional affiliations.

- fast, convenient online submission

- thorough peer review by experienced researchers in your field

- rapid publication on acceptance

- support for research data, including large and complex data types

- gold Open Access which fosters wider collaboration and increased citations

- maximum visibility for your research: over $100 \mathrm{M}$ website views per year

At BMC, research is always in progress.

Learn more biomedcentral.com/submissions 